\title{
The efficacy and safety of epidural oxycodone in pain management after gynaecologic laparoscopy
}

\author{
Panu Piirainen ${ }^{1,2}$, Hannu Kokki ${ }^{1,3}$, Heidi Hautajärvi ${ }^{4}$, Veli-Pekka Ranta ${ }^{5}$, Merja Kokki ${ }^{3}$ \\ ${ }^{1}$ School of Medicine, Faculty of Health Sciences, University of Eastern Finland, Kuopio, Finland \\ ${ }^{2}$ Department of Anaesthesia and Intensive Care, Kajaani Central Hospital, Kajaani, Finland \\ ${ }^{3}$ Department of Anaesthesia and Operative Services, Kuopio University Hospital, Kuopio, Finland \\ ${ }^{4}$ Admescope Ltd, Oulu, Finland \\ ${ }^{5}$ School of Pharmacy, Faculty of Health Sciences, University of Eastern Finland, Kuopio, Finland
}

\section{Background}

Early pain after laparoscopic surgery is often moderate to severe (1). Epidural oxycodone penetrates readily into cerebrospinal fluid and may be more potent than intravenous (i.v.) oxycodone (2). In this randomised, double blind clinical trial, we have assessed the analgesic efficacy of epidural oxycodone after gynaecologic laparoscopy.

\section{Methods}

We enrolled 60 women, aged 23-71 years, undergoing elective gynaecologic laparoscopy under standardized general anaesthesia.

Postoperatively, the patients were administrated either i.v. saline and epidural oxycodone $0.1 \mathrm{mg} \cdot \mathrm{kg}^{-1}$ (EPI-group, $\mathrm{n}=31$ ) or i.v. oxycodone $0.1 \mathrm{mg} \cdot \mathrm{kg}^{-1}$ and epidural saline (IV-group, $n=29$ ). For background analgesia the subjects received i.v. paracetamol and dexketoprofen (a NSAID). The primary outcome was the amount of i.v. fentanyl for rescue analgesia during the first four postoperative hours.

Analysis of continuous data were performed with the two sample t-test or the Mann-Whitney U-test. Categorical data were analysed using the ChiSquare test.

\section{Results}

The patient characteristics are presented in table.

The median amount of fentanyl for rescue analgesia during the first four postoperative hours was 50 (0450) $\mu \mathrm{g}$ in the EPI-group and $100(0-550) \mu \mathrm{g}$ in the IV-group, $p=0.025$.

More patients had pruritus in the EPI-group than in the IV-group, 17 vs. 8 , respectively, $p=0.032$.

\section{Conclusion}

Analgesic efficacy of epidural oxycodone was superior compared to that of i.v. administration in early pain management after laparoscopic surgery

Table. Patient characteristics. Data are mean (standard deviation) and number of cases.

\begin{tabular}{|l|c|c|c|}
\hline Variable & $\begin{array}{c}\text { EPI-group } \\
\mathbf{n = 3 1}\end{array}$ & $\begin{array}{c}\text { IV-group } \\
\mathbf{n = 2 9}\end{array}$ & p-value \\
\hline Age, yr & $52(12)$ & $53(13)$ & 0.16 \\
\hline Weight, kg & $72(13)$ & $74(15)$ & 0.65 \\
\hline Height, cm & $162(4.9)$ & $165(6.9)$ & 0.080 \\
\hline BMI, kg m-2 & $27(4.3)$ & $27(4.7)$ & 0.95 \\
\hline ASA, I/II/III & $6 / 21 / 4$ & $7 / 18 / 4$ & 0.89 \\
\hline $\begin{array}{l}\text { Duration of } \\
\text { surgery, min }\end{array}$ & $210(98)$ & $220(111)$ & 0.88 \\
\hline $\begin{array}{l}\text { Intraoperative } \\
\text { bleeding, } \mathrm{mL}\end{array}$ & $270(370)$ & $290(510)$ & 0.16 \\
\hline
\end{tabular}

\section{References}

1. Sjövall S, Kokki M, Kokki H. Laparoscopic surgery: a narrative review of pharmacotherapy in pain management. Drugs. 2015;75(16):1867-89.

2. Kokki M, Välitalo P, Kuusisto M, et al. Central nervous system penetration of oxycodone after intravenous and epidural administration. Br J Anaesth 2014; 112(1): 133-40.

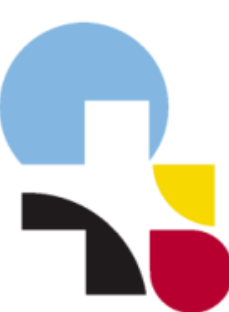

Kuopio

University

Hospital 